\title{
Microwave switchable frequency selective surface with high quality factor resonance and low polarization sensitivity
}

\author{
Victor Dmitriev and Marcelo N. Kawakatsu \\ Department of Electrical Engineering, Federal University of Para, Belem-PA, Brazil, victor@ufpa.br and \\ mnkawakatsu@yahoo.com.br
}

\begin{abstract}
We present a microwave switchable frequency selective surface with high quality factor transmission resonance. The high quality resonance is achieved by excitation of the trapped-mode in array with two concentric metal rings in a cell on a silicon substrate. Optical activation of the silicon substrate permits to switch off the transmission band.
\end{abstract}

Index Terms - Frequency selective surface, high quality factor resonance, trapped-mode resonance, optical activation.

\section{INTRODUCTION}

Control of microwave propagation by a boundary surface or a layer is a subject of intense investigation in applied electrodynamics. Arrays of patches or aperture elements can have a great versatility of electromagnetic responses. Unusual applications of these arrays in microwave region include invisibility metal [1], magnetic mirror [2-3], optical activity [4], thin electromagnetic absorbers [5] and reconfigurable antennas [6]. In terahertz region, applications related to wave manipulation and sensing have been also reported [7-8].

In this work we propose a switchable FSS with high quality factor (Q-factor) transmission resonance controllable by optical activation of its silicon substrate. This structure can be used in antennas and waveguide technologies, and in electromagnetic compatibility systems, for example, in active filters. The high Q-factor resonance is achieved by excitation of the so-called trapped-mode regime in array where every cell is formed by two concentric rings. This array pattern was originally proposed in [9].

The trapped-mode is defined by excitation of anti-symmetric currents in the rings. This mode is weakly coupled to free-space, therefore the radiation losses are low and the Q-factor is very high as compared with a conventional resonance mode [10]. The Q-factor of presented FSS is around 8 while that of a similar FSS with a single ring resonant element is around 1 (we define the Q-factor as the ratio between the resonance frequency and the full width at half maximum of transmission).

\section{PROBLEM DESCRIPTION}

The FSS consists of a periodic array of two concentric metal rings in a square cell and supported by a silicon substrate as depicted in Fig. 1. Also, we show in this figure the projections of the wave number $\boldsymbol{k}_{x y}$ and $\boldsymbol{k}_{x z}$ of the incident plane wave on the $x y$ - and $x z$-planes, respectively. Due to high 
rotational symmetry, this array is polarization independent for normal incidence [11]. We have chosen the dimensions of the array shown in Fig. 1 in accordance with the results of [12], where a study of the influence in trapped-mode resonance with substrate loss and rings dimensions was made. The following values of geometrical parameters were considered in the simulations for obtaining a trapped-mode resonance around $40 \mathrm{GHz}: D_{x}=D_{y}=1.3, r_{o}=0.6, r_{i}=0.5, w=0.02$ and $h=0.05$ (all dimensions in $\mathrm{mm})$.

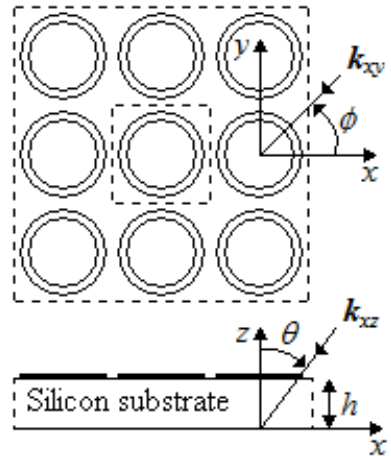

(a)

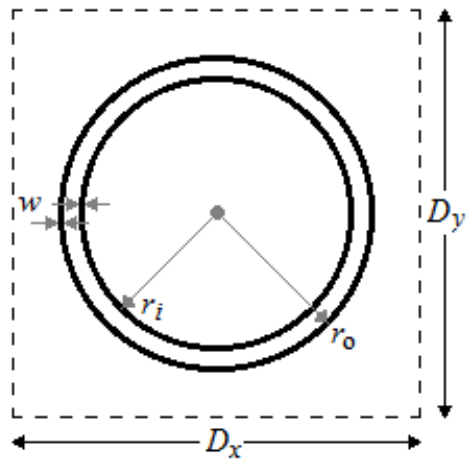

(b)

Fig. 1. FSS consisting of array of two concentric rings supported by silicon substrate (a) and unit cell of the array (b).

In our simulations the array of metal rings made of perfect conductors is considered to be infinite in $x$ - and $y$-directions. The optically activated silicon substrate was modeled as in [13], in which the relative permittivity of the silicon can be described in the form

$$
\varepsilon_{r}=\varepsilon_{p}-\frac{\omega_{p}^{2}}{\omega^{2}+v^{2}}-j \frac{v}{\omega} \frac{\omega_{p}^{2}}{\omega^{2}+v^{2}}
$$

where $\varepsilon_{p}$ is the dielectric constant of the silicon in passive state, $\omega_{p}^{2}=N e^{2} / \varepsilon_{0} m^{*}$ is the plasma frequency, $v$ is the collision angular frequency, $N$ is the plasma density, $e$ is the electron charge, $m *$ is the effective mass of the charge carrier and $\varepsilon_{0}$ is the free-space permittivity. Since the optically activated silicon substrate behaves as a semiconductor containing electron-hole pairs, it is necessary in Eq. (1) to take into account the contributions of electrons and holes separately as described in [13]. Using the same numerical values given in [13], we calculated the behavior of the complex refractive index $n$ as a function of the plasma density $N$ for the frequency of $40 \mathrm{GHz}$, as can be seen in Fig. 2 . 


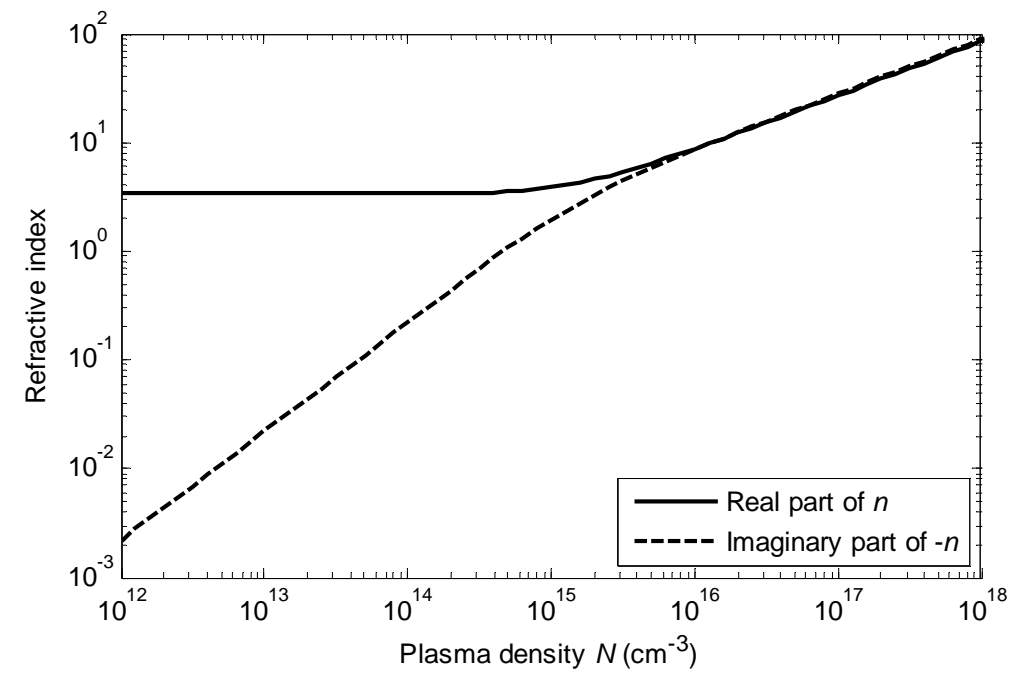

Fig. 2. Refractive index of the silicon substrate as a function of the plasma density $N$.

\section{NUMERICAL RESULTS}

For numerical calculation of the reflection and transmission characteristics, we used the wellestablished spectral domain moment method (SDMM) which consists in solving by method of moments the integral equation of the problem in the frequency domain [14]. In this method, the periodic boundary conditions are taken into account by considering only harmonics of the components $k_{\mathrm{x}}$ and $k_{\mathrm{y}}$ of the wavenumber which follows from the periodicities of the array in the $\mathrm{x}$ - and $\mathrm{y}$ directions. The substrate was modeled by the Green's function obtained by applying the boundary conditions on the top and the bottom interfaces of the substrate. The surface current density on the rings was modeled using the roof-top subdomain basis functions. For validating our results, we used the commercial software Computer Simulation Technology (CST) [15], which is based on the finite integration technique.

In Fig. 3 are shown the reflection, transmission and normalized absorption frequency responses for $N=10^{14} \mathrm{~cm}^{-3}$ calculated using SDMM and CST, demonstrating good agreement between the results. In Fig. 4 the reflection and transmission curves of the array for normally incident plane wave and different values of $N$ are shown. These graphics were obtained with SDMM. For $N=10^{12} \mathrm{~cm}^{-3}$, the influence of plasma on the refraction index of the silicon substrate is negligible and a high Q-factor transmission resonance exists with almost total transmission at $40 \mathrm{GHz}$. Note that the trapped-mode resonance is between two broad reflection resonances, which are generated by the outer and inner rings. Increasing $N$ to $10^{18} \mathrm{~cm}^{-3}$, one comes to the regime of almost complete reflection of the incident wave due to the significant increase of the real and imaginary parts of the silicon refraction index (see Fig. 2). 


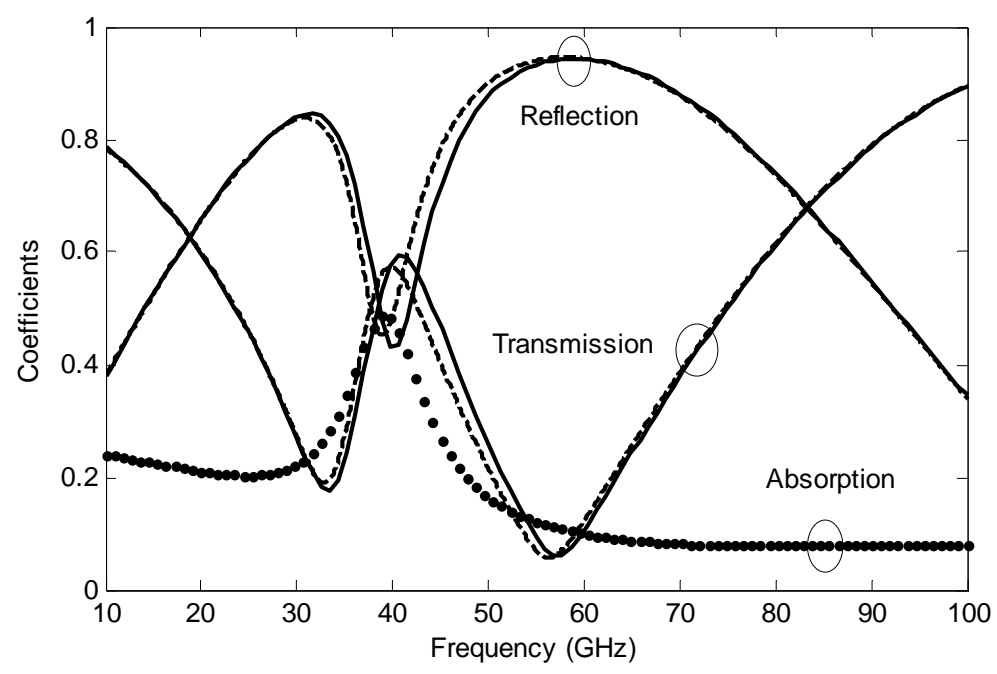

Figure 3. Reflection and transmission frequency responses of FSS for normal incidence and $N=10^{14} \mathrm{~cm}^{-3}$, calculated using SDMM (solid-curve and dotted-curve) and CST (dashed-curve).

In Fig. 5 the reflection and transmission curves are shown for inclined incidence with $\phi=0^{\circ}, \theta=$ $30^{\circ}, N=10^{12} \mathrm{~cm}^{-3}$ and for TE- or TM-polarizations. For the TE case, the Q-factor of the trappedmode resonance is a little bit higher and the adjacent reflection resonances are broadened in comparison with the normal incidence. For the TM case, the opposite behavior is observed. The small peaks and deeps near $80 \mathrm{GHz}$ are due to grating lobes. Our analysis shows that due to the high rotational symmetry of the array unit cell, the responses are almost insensitivity to the angle $\phi$.

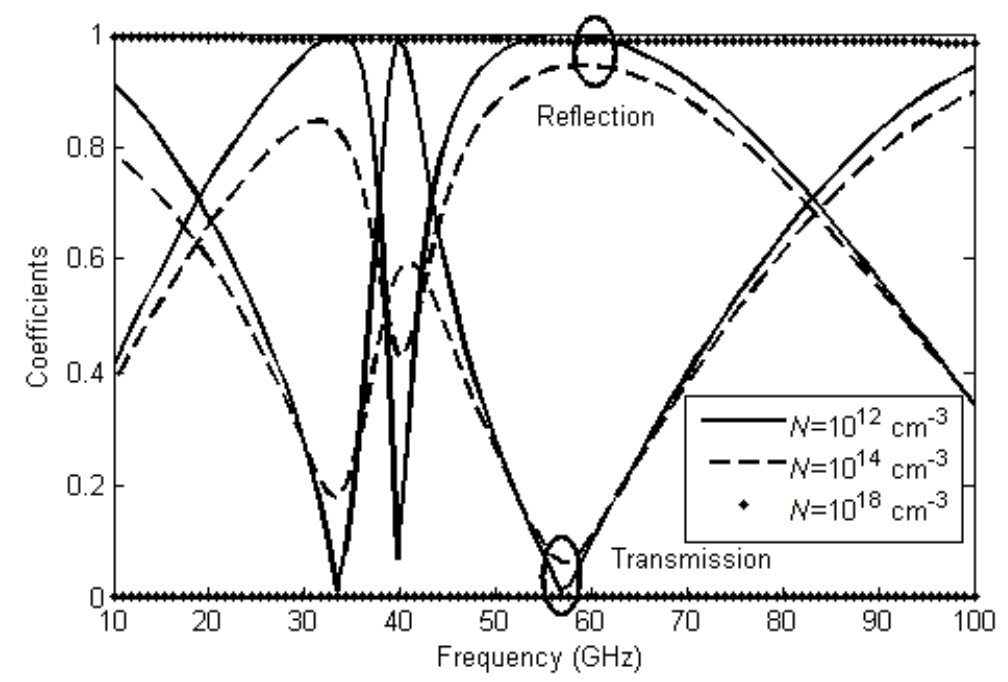

Figure 4. Reflection and transmission frequency responses of FSS for normal incidence and different values of $N$. 


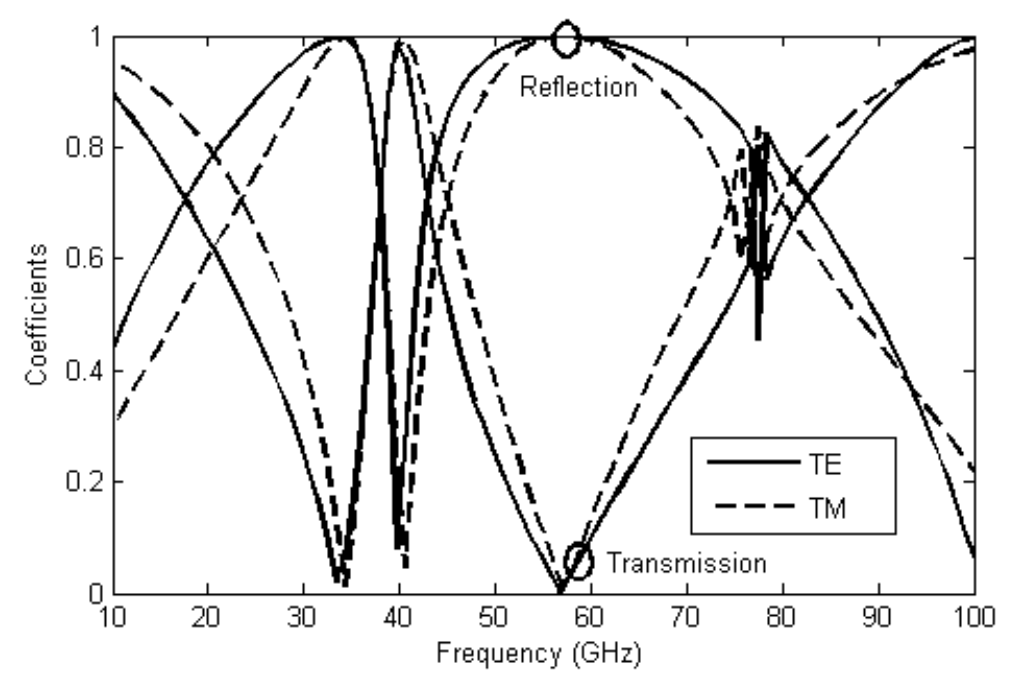

Figure 5. Reflection and transmission frequency responses of FSS for incidence with $\phi=0^{\circ}$ and $\theta=30^{\circ}, N=10^{12} \mathrm{~cm}^{-3}$, and TE- or TM-polarization.

\section{CONCLUSION}

We suggested and analyzed a switchable FSS with high Q-factor transmission resonance and low polarization sensitivity. The analyzed FSS has the Q-factor about 8, which is much higher than that of similar FSS with a single ring resonant element. High Q-factor is achieved due to excitation of the trapped-mode. Using an optically activated silicon substrate, we demonstrated theoretically that it is possible to switch on and off the transmission band. The frequency band of the structure can be scaled to lower or higher frequencies.

\section{ACKNOWLEDGMENTS}

This work was financially supported by the Brazilian agencies CNPq and CAPES.

\section{REFERENCES}

[1] V. A. Fedotov, P. L. Mladyonov, S. L. Prosvirnin, and N. I. Zheludev, "Planar electromagnetic metamaterial with a fish scale structure”, Phys. Rev. E, vol. 72, November 2005.

[2] D. Sievenpiper, L. Zhang, R. F. J. Broas, N. G. Alexopolous, E. Yablonovitch, "High-impedance electromagnetic surfaces with a forbidden frequency band”, IEEE Trans. Microw. Theory Tech., vol. 47, issue 11, pp. 2059-2074, November 1999.

[3] M. Hosseini, A. Pirhadi, and M. Hakkak, "A novel AMC with little sensitivity to the angle of incidence using 2-layer Jerusalem Cross FSS”, Progress In Electromagnetics Research, vol. 64, pp. 43-51, 2006.

[4] E. Plum, V. A. Fedotov, and N. I. Zheludev, "Optical activity in extrinsically chiral metamaterial”, App. Phys. Lett., vol. 93, issue 19, 2008.

[5] F. Costa, A. Monorchio, and G. Manara, "Analysis and Design of Ultra Thin Electromagnetic Absorbers Comprising Resistively Loaded High Impedance Surfaces”, IEEE Trans. Antennas Propagat., vol.58, no.5, pp.1551-1558, May 2010.

[6] J. Constantine, "Design, optimization and analysis of reconfigurable antennas”, PhD Dissertation, University of New Mexico, December 2009.

[7] H. -T. Chen, W. J. Padilla, J. M. O. Zide, A. C. Gossard, A. J. Taylor, and R. D. Averitt, “Active terahertz metamaterial devices”, Nature, vol. 444, pp. 597-600, November 2006.

[8] R. Singh, E. Smirnova, A. J. Taylor, J. F. O'Hara, and W. Zhang, “Optically thin terahertz metamaterials”, Optics Express, vol. 16, issue 9, pp. 6537-6543, 2008. 
[9] N. Papasimakis, Y. H. Fu, V. A. Fedotov, S. L. Prosvirnin, D. P. Tsai, and N. I. Zheludev, "Metamaterial with polarization and direction insensitivity resonant transmission response mimicking electromagnetically induced transparency”, Appl. Phys. Lett., vol. 94, 211902, 2009.

[10] V. A. Fedotov, M. Rose, S. L. Prosvirnin, N. Papasimakis, and N. I. Zheludev, "Sharp Trapped-Mode Resonance in Planar Metamaterials with a Broken Structural Symmetry”, Phys. Rev. Lett., vol. 99, issue 14, October 2007.

[11] V. Dmitriev, "Symmetry properties of electromagnetic planar arrays: Long-wave approximation and normal incidence”, Metamaterials Congress 2010, vol. 5, issue 2-3, pp. 141-148, September 2011.

[12] M. N. Kawakatsu, V. A. Dmitriev, and S. L. Prosvirnin, "Microwave Frequency Selective Surfaces with High Q-Factor Resonance and Polarization Insensitivity”, Journal of Electromagnetic Waves and Applications, vol. 24, pp. 261-270, 2010.

[13] C. H. Lee, P. S. Mak, and A. P. De Fonzo, "Optical Control of Millimeter-Wave Propagation in Dielectric Waveguides”, IEEE J. Quantum Electron., vol. E-16, no. 3, March 1980.

[14] R. Mittra, C. H. Chan and T. Cwik, "Techniques for Analyzing Frequency Selective Surfaces - A Review", Proceedings of the IEEE, pp. 1593-1615, 1988.

[15] www.cst.com. 